\title{
Poor adherence to the World Health Organization guidelines of treatment of severe pneumonia in children at Khartoum, Sudan
}

\author{
Karim Eldin M Salih', Jalal A Bilal ${ }^{2}$, Mona A Alfadeel ${ }^{3}$, Yassin Hamid ${ }^{4}$, Widad Eldouch', Elfatih Elsammani ${ }^{6}$, \\ Salah A Ibrahim and Ishag Adam $2,7^{*}$
}

\begin{abstract}
Background: Community-acquired pneumonia (CAP) is as a major cause for childhood morbidity and mortality worldwide. This study was conducted to investigate the adherence and response of the WHO guidelines for treatment of severe pneumonia.

Method: A cross-sectional study was conducted in the period of June 2009 to July 2010 at Khartoum Hospital, Sudan. Children admitted and treated for severe pneumonia were enrolled.

Results: Only 39 (18.8\%) out of 208 enrolled children received prescriptions that were adherent to the WHO guidelines of treatment of severe pneumonia. In logistic regression none of the investigated variable (age, gender, and clinical presentations) was associated with the adherence to the WHO guidelines. There was no significant difference in the response between adherent and non-adherent prescriptions. There was no association between the demographic, clinical data, treatment-adherence to the guidelines and the patients' response.
\end{abstract}

Conclusion: There is a poor (18.8\%) adherence to the WHO guidelines of the treatment of severe pneumonia in the region regardless to the age, gender and clinical presentation.

Keywords: Pneumonia, Treatment, Adherence, Penicillin, Sudan

\section{Introduction}

Community-acquired pneumonia (CAP) is as a major cause for childhood morbidity and mortality worldwide [1]. World Health Organization has estimated an annual global incidence of new episodes of pneumonia as156 million, the vast majority of these (151 million) episodes were reported from the developing countries [2]. The leading bacterial cause of pneumonia in underdeveloped countries is pneumococcus (30-50\%), H. influenzae type b (10-30\% of cases), S. aureus and K. pneumoniae [3]. Empiric antibacterial selection for treatment of childhood CAP must be based on age and the likely causative organisms [4]. Early and prompt diagnosis of bacterial pneumonia is lacking in most of the developing countries, hence empirical treatment is usually practiced.

\footnotetext{
* Correspondence: ishagadam@hotmail.com

${ }^{2}$ College of Medicine, Qassim University, Buraydah, Saudi Arabia

${ }^{7}$ Faculty of Medicine, University of Khartoum, Khartoum, Sudan

Full list of author information is available at the end of the article
}

Sudan has one of the highest infant mortality rates (112/ 1000 live birth) and respiratory infections are among the most killing causes [5]. There is no published data addressing pneumonia/treatment in Sudan. Most hospital guidelines in developing countries recommend the use of penicillin and its derivatives for treatment of severe pneumonia based on the WHO guidelines [6]. Generally, many clinicians are concerned that guidelines do not reflect the complexity of the real situation and may stand against those who advocate its application in clinical practice [7].

The objectives of this study were to determine the adherence to the $\mathrm{WHO}$ guidelines for treatment of severe pneumonia and to compare the response to the treatment between the adherences and an empirical antibiotic regimen initially used in the treatment of patients with severe CAP at the end of 48 hours of starting antibiotic in a major pediatric facility in Sudan.

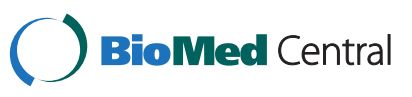

(c) 2014 Salih et al.; licensee BioMed Central Ltd. This is an Open Access article distributed under the terms of the Creative Commons Attribution License (http://creativecommons.org/licenses/by/4.0), which permits unrestricted use, distribution, and reproduction in any medium, provided the original work is properly credited. The Creative Commons Public Domain Dedication waiver (http://creativecommons.org/publicdomain/zero/1.0/) applies to the data made available in this article, unless otherwise stated. 


\section{Methods}

A longitudinal hospital-based study was conducted at the Children Emergency Hospital (CEH), Khartoum, Sudan in the period from June 2009 to July 2010. CEH is the main urban children referral hospital in Sudan with the capacity of 240 beds and a catchment population of more than five million people representing the population of the tripartite metropolis, Khartoum [8].

After signing an informed consent (by the parents/ guardian) all consecutively admitted children aged 2 to 59 month with definite labeling diagnosis of "severe pneumonia", based on the WHO criteria for diagnosis of pneumonia were included [6]. The critically ill children, children with known TB, HIV, bronchial asthma, other chronic conditions that might be complicated by pneumonia and those who refuse to be enrolled in the study were excluded. A radiologist reviewed the chest radiographs (CXR) taken for every child satisfying the WHO criteria for severe pneumonia at the time of admission. Only those with evidence of lobar or segmental consolidation or with an infiltrate in at least one lung on chest radiographs were included.

The investigators recorded demographic, clinical and treatment data as prescribed and written by the treating physicians from patients files at the time of admission and at day 2 to determine response or failure to the antibacterial treatment. Evaluation data include symptoms (cough, inability to drink, vomiting, lethargy), clinical signs (tachypnea, temperature of $\geq 37.5^{\circ} \mathrm{C}$, signs of respiratory distress, grunting and full chest examination). A patient was labeled "responding" if the respiratory rate returned to age specific normal range, less fever and eating better after 48 hours following prescription [6]. The patient was labeled "not responding" if respiratory rate did not return to age-specific normal at the end of 48 hours of antibiotic treatment, still febrile and/not eating better.

\section{Ethical consideration}

The Development and Ethics Committee of Khartoum Children Emergency Hospital approved this study prior to collecting data.

\section{Statistical analyses}

Data were entered and analyzed into SPSS statistical software version 16.0 (SPSS Inc., Chicago, IL, U.S.A. 2007). The descriptive analyses used included the mean, standard deviation, and frequency distribution. Chi square test was used to compare the difference between categorical data. Univariate and multivariate analyses were performed where adherence (penicillin prescription) was the dependent variable and the other variables (age, gender, symptoms) were the independent variable. Another model was performed for the outcome of treatment. Odds ration and 95\% confidence interval (CI) were calculated and $\mathrm{P}<0.05$ was considered significant.

\section{Results}

Two hundred and eight children fulfilled the inclusion criteria in this study. Table 1 shows the demographic and clinical characteristics of the studied group. The mean (SD) of the age was 28.12 (13.9) months and 99 (47.6\%) were males. Only $39(18.8 \%)$ of these children received the prescription that was adherent to the WHO guidelines (penicillin) of severe pneumonia.

The antimicrobial prescriptions, which were not in accordance with the WHO guidelines, were: amoxicillin/ clavulanic acid $(46,22.1 \%)$, ceftriaxone $(42,20.2 \%)$, cefuroxime $(41,19.7 \%)$, a combination of penicillin/gentamicin (29; $13.9 \%)$ and others $11(11,5.3 \%)$.

None of the investigated factors (age, gender, symptoms and signs) were found as predictors for adherence of the WHO guidelines for treatment of severe pneumonia, Table 2.

There was no significant difference in the response between adherence and non-adherence prescriptions [32/39 (82.1\%) vs. $151 / 169$ (81.2\%), P = 0.206]. None of the investigated predictors was found to be associated with treatment outcome as shown, Table 3.

\section{Discussion}

The WHO has stressed on the accountability and accessibility of the health information and data at the country level to measure and monitor results. The data collection on children health is essential to determine for focusing of investments and progress monitoring [9]. The main findings in this study were that there was poor adherence to the WHO guidelines of treatment of severe pneumonia in under-five year old children. The almost $82 \%$ of non-adherence to the WHO guidelines of severe pneumonia in children indicated that patients did not receive the appropriate medication to their clinical needs and the antibiotics were not at the lowest cost to them and their community. This almost defines an irrational use of antibiotics [10]. The Sudan Association of

Table 1 Clinical manifestation of the children

\begin{tabular}{ll}
\hline Variable & Frequency (\%) \\
\hline Fever & $196(94.2)$ \\
Active alae nasi & $175(93.1)$ \\
Intercostal recession & $174(92.6)$ \\
Shortness of breathing & $192(92.3)$ \\
Cough & $181(88.3)$ \\
Runny nose & $158(85.4)$ \\
Chest indrawing & $154(81.9)$ \\
Grunting & $135(73.4)$ \\
\hline
\end{tabular}


Table 2 Factors associated with adherence to WHO guidelines of treatment of severe pneumonia in the study using univariate analyses

\begin{tabular}{lll}
\hline Variables & OR $(\mathbf{9 5} \% \mathbf{C l})$ & P value \\
\hline Age & $0.9(0.9-1.1)$ & 0.902 \\
Sex & $1.3(0.6-2.81)$ & 0.380 \\
Fever & $0.8(0.1-4.0)$ & 0.849 \\
Cough & $0.8(0.2-26.9)$ & 0.530 \\
Runny nose & $0.9(0.4-2.2)$ & 0.913 \\
Breathlessness & $0.5(0.1-2.7)$ & 0.742 \\
Grunting & $0.8(0.3-1.9)$ & 0.838 \\
Active alae nasi & $1.1(0.3-3.7)$ & 0.761 \\
Intercostal recession & $1.8(0.5-6.1)$ & 0.304 \\
Chest indrawing & $1.1(0.4-2.8)$ & 0.645 \\
\hline
\end{tabular}

Paediatricians developed a local WHO-based protocol for treatment of emergency conditions in children including pneumonia in 2011. Though this protocol was not validated and did not take effect in clinical practice, yet the adherence in this study was not in compliance with its treatment guidelines [11]. The non-adherence rate was higher than that reported from Burkina Faso. Their estimate was around $61 \%$ though it was an estimate of compliance for pneumonia among other diseases yet it is here as well [12]. Moreover the prescribing personnel, in their report, were nurses and health workers and not physicians as in our study. In a Tanzanian study, half of the children with severe pneumonia were prescribed antibiotics, which are not recommended by the WHO [13]. Even in developed countries, the compliance to privileged countries guidelines is not usually perfect despite good training, a fact, which was illustrated in one study where even, restricted initial broad-spectrum

Table 3 Factors associated with outcome of treatment of severe pneumonia in children in the study using univariate and multivariate analyses

\begin{tabular}{|c|c|c|c|c|}
\hline \multirow[t]{2}{*}{ Variables } & \multicolumn{2}{|l|}{ Univariate } & \multicolumn{2}{|l|}{ Multivariate } \\
\hline & OR $(95 \% \mathrm{Cl})$ & $\mathbf{P}$ & OR $(95 \% \mathrm{Cl})$ & $\mathbf{P}$ \\
\hline Adherence & $0.5(0.2-1.4)$ & 0.271 & $2.3(0.8-6.6)$ & 0.099 \\
\hline Age & $0.8(0.18-4.09)$ & 0.849 & $0.9(0.9-1.1)$ & 0.255 \\
\hline Sex & $0.8(0.2-2.6)$ & 0.530 & $0.8(0.3-2.1)$ & 0.701 \\
\hline Clinical & $0.9(0.4-2.2)$ & 0.913 & $1.0(0.3-3.4)$ & 0.925 \\
\hline Cough & $0.9(0.2-3.5)$ & 0.961 & $1.2(0.2-5.8)$ & 0.753 \\
\hline Runny nose & $2.9(1.2-7.1)$ & 0.017 & $0.3(0.1-1.4)$ & 0.065 \\
\hline Breathlessness & $2.1(0.2-16.9)$ & 0.470 & $0.4(0.1-4.0)$ & 0.479 \\
\hline Grunting & $0.6(0.2-1.3)$ & 0.429 & $1.6(0.5-4.9)$ & 0.376 \\
\hline Active alae nasi & $1.1(0.2-5.4)$ & 0.834 & $0.7(0.9-5.7)$ & 0.770 \\
\hline Intercostal recession & $1.8(0.2-14.6)$ & 0.567 & $0.3(0.1-2.9)$ & 0.362 \\
\hline Chest indrawing & $2.0(0.2-15.9)$ & 0.512 & $2.2(0.5-4.9)$ & 0.116 \\
\hline
\end{tabular}

antimicrobial therapy to cultured pathogens was a rare practice [14]. The statement of the guidelines might not be clear, especially for junior physicians as well as it was not endorsed by the senior physicians who used to train them. Tis might, possibly be, because they were not convinced of the recommended antibiotic in the guidelines however, studies on knowledge attitude and behavior towards formal guidelines may well explain this point.

The fact that there was no difference in treatment outcome when following the WHO guidelines or using alternative antibiotics, in this study, may serve adherence to the guidelines until prove otherwise. A similar finding was reported in a Kenyan survey where poor guideline adherence was attributed to preferences for broader spectrum, non-beta-lactam antibiotics [15]. Moreover, another Kenyan study reported a lower rate (48.3\%) among children with pneumonia in which prescription had improved to $90 \%$ adherence after intervention (training and booklet distribution) [16]. This praises training as an important tool in improving adherence and thus better outcome of pneumonia in children.

Antibiotics prescribed in this study except for penicillin do not follow any of the international guidelines. Commonly used antibiotics for severe pneumonia in an African setting were penicillin, sulphonamides, and aminoglycosides [17]. Amoxicillin and sulphonamides were the commonly encountered prescriptions in management of pneumonia in other areas too e.g. Tanzania and rural Peru $[12,18]$. The results of a Sudanese survey in prescribing patterns proved that at least some parts of population are unable to afford treatment for some important and common diseases including childhood pneumonia and an average $66 \%$ rate of antibiotics prescription [19]. The variable antibiotic prescription may depend not only on guidelines unacceptability among physicians but also on health provider characteristics, experience and attitude [15]. Moreover, the health facilities settings and equipment may be an important factor. This study showed no correlation between clinical presentation and treatment outcome of severe pneumonia in children. However, duration of fever, rather than mere fever and tachypnea were documented as strong predictors for diagnosis of pneumonia rather than outcome in one study [20]. Another study reported tachypnea and chest indrawing as best predictors of pneumonia severity [21]. An extended evaluation in further studies is amenable to assess the morbidity and mortality of guidelinebased treatment pneumonia in this age group.

This study did not address the health system, physician and family factors that may influence the implementation of the guidelines and the long-term outcome of children such as mortality. It was not the aim of this work, albeit useful, to study the cure rate, the full recovery and mortality of the children with severe 
pneumonia. Moreover, prior antibiotic treatment before admission was not recorded as well as supportive therapy. However this study, to the best of our knowledge, is the first of its type to determine physicians' adherence to health policies in Sudan. The relatively reasonable sample in this study enabled us to draw conclusions on the adherence to the widely practiced $\mathrm{WHO}$ guidelines and the pattern of antibiotic prescription in a developing country.

\section{Conclusion}

The non-adherence to WHO guidelines of severe pneumonia is the highest in the region $(82 \%)$ regardless to the age and gender. There was no difference in the response between adherence and non-adherence. Studies are needed to determine physician and family factors influencing the implementation of the guidelines and their effects on the long term.

\section{Competing interest}

Authors declare that they have no conflict of interest

\section{Authors' contributions}

KMS, MAA, WE and SAI collected data. JAB, YH, EE and IA contributed to the data analysis and statistical analysis. All authors have read the manuscript to revise it carefully and have approved the final manuscript.

\section{Acknowledgement}

The authors would like to thank the children and their parents who participated in this study. Our sincere appreciation goes to Dr. Osman Abdalwahab, senior consultant radiologist, Ministry of Health, Sudan for his valuable comments on $\mathrm{X}$ rays as well as for the laboratory and the nursing staff of Khartoum Children Hospital for their contribution.

\section{Author details}

'Department of Pediatrics, College of Medicine, King Khalid University, Abha, Saudi Arabia. ${ }^{2}$ College of Medicine, Qassim University, Buraydah, Saudi Arabia. ${ }^{3}$ King Khalid University Hospital, Riyadh, Saudi Arabia. ${ }^{4}$ Almaarifa College for Science and Technology, Khartoum Children, Riyadh, Saudi Arabia. ${ }^{5}$ Department of epidemiology, Federal Ministry of Health, Khartoum, Sudan. ${ }^{6}$ Department of Epidemiology, School of Medicine, Ahfad University for Women, Omdurman, Sudan. ${ }^{7}$ Faculty of Medicine, University of Khartoum, Khartoum, Sudan.

Received: 27 July 2014 Accepted: 11 August 2014

Published: 14 August 2014

\section{References}

1. British Thoracic Society Standards of Care Committee: BTS Guidelines for the Management of Community Acquired Pneumonia in Childhood. Thorax 2002, 57(Suppl. 1):i1-i23.

2. Rudan I, Boschi-Pinto C, Biloglav Z, Mulholland K, Campbell H: Epidemiology and etiology of childhood pneumonia. Bull World Health Organ 2008, 86:408-416.

3. Manikam L, Lakhanpaul M: Epidemiology of community acquired pneumonia. J Paediatr Child Health 2012, 22:299-306.

4. Korppi M: Community-acquired pneumonia in children: issues in optimizing antibacterial treatment. Paediatr Drugs 2003, 5:821-832.

5. World Health Organization: Country Cooperation Strategy for WHO and Sudan, 2008-2013. Available at: http://www.who.int/countryfocus/ cooperation_strategy/ccs_sdn_en.pdf.

6. WHO: Pocket book of hospital care for children: quidelines for the management of common illnesses with limited resources. Geneva: World Health Organization; 2005. http://www.who.int/maternal_child_adolescent/ documents/child_hospital_care/en/ (accessed Oct 21, 2013).
7. Garfield FB, Garfield JM: Clinical judgment and clinical practice guidelines. Int J Technol Assess Health Care 2000, 16:1050-1060.

8. The fifth population census data: Sudan Central Bureau of statistics. 2008 Available from: http://www.cbs.gov.sd/en/.

9. Commission on Information and Accountability for Women's and Children's Health: Keeping promises, measuring results. 2011. http://www. everywomaneverychild.org/images/content/files/accountability_commission/ final_report/Final_EN_Web.pdf. Accessed 4 December 2013.

10. WHO: The Rational Use of Drugs - Report of the Conference of Experts, Nairobi 25-29 November 1985. Geneva: World Health Organization; 1987.

11. Sudanese Association of Paediatricians: Management Protocols for Paediatric Emergency. Sudan Association of Paediatricians and Advisory committee of paediatricians. 2nd edition. 2011:32-33. Available from: http://www.sudanjp. org/uploads/9/2/7/0/9270568/paediatric_emergency_guidelines.pdf.

12. Ilboudo TB, Chou Y, Huang N: Assessment of providers' referral decisions in Rural Burkina Faso: a retrospective analysis of medical records. BMC Health Serv Res 2012, 12:54

13. Gwimile JJ, Shekalaghe SA, Kapanda GN, Kisanga ER: Antibiotic prescribing practice in management of cough and/or diarrhea in Moshi Municipality, Northern Tanzania: cross-sectional descriptive study. Pan Afr Med J 2012, 12:103.

14. Mol PG, Denig P, Gans RO, Nannanpanday PV, Degener JE, Laseur M, Haaijer-Ruskamp FM: Limited effect of patient and disease characteristics on compliance with hospital antimicrobial guidelines. Eur J Clin Pharmacol 2006, 62:297-305

15. English M, Esamai F, Wasunna A, Were F, Ogutu B, Wamae A, Snow RW, Peshu N: Delivery of paediatric care at the first-referral level in Kenya. Lancet 2004, 364:1622-1629.

16. Irimu GW, Gathara D, Zurovac D, Kihara H, Maina C, Mwangi J, Mbori-Ngacha D, Todd J, Greene A, English M: Performance of Health Workers in the Management of Seriously Sick Children at a Kenyan Tertiary Hospital: Before and after a training intervention. PLoS One 2012, 7(7):e39964.

17. Krause G, Borchert M, Benzler J, Diesfeld HJ: From diagnosis to drug taking: staff compliance with guidelines and patient compliance to prescriptions in Burkina Faso. Int J Qual Health Care 2000, 12(1):25-30.

18. Kristiansson C, Reilly M, Gotuzzo E, Rodriguez H, Bartoloni A, Thorson A, Falkenberg T, Bartalesi F, Tomson G, Larsson M: Antibiotic use and health-seeking behaviour in an underprivileged area of Peru. Trop Med Int Health 2008, 13:434-441.

19. Cheraghali AM, Idries AM: Availability, affordability, and prescribing pattern of medicines in Sudan. Pharm World Sci 2009, 31:209-215.

20. Nijman RG, Vergouwe $Y$, Thompson $M$, Van Veen $M$, Van Meurs AH, van der Lei J, Steyerberg EW, Moll HA, Oostenbrink R: Clinical prediction model to aid emergency doctors managing febrile children at risk of serious bacterial infections: diagnostic study. BMJ 2013, 346:f1706.

21. Nacul LC, Kirkwood BR, Carneiro AC, Pannuti CS, Magalhaes M, Arthur PA: Etiology and clinical presentation of pneumonia in hospitalized and outpatient children in Northeast Brazil and risk factors for severity. $J$ Health Popul Nutr 2005, 23:6-15.

doi:10.1186/1756-0500-7-531

Cite this article as: Salih et al.: Poor adherence to the World Health Organization guidelines of treatment of severe pneumonia in children at Khartoum, Sudan. BMC Research Notes 2014 7:531.

\section{Submit your next manuscript to BioMed Central and take full advantage of:}

- Convenient online submission

- Thorough peer review

- No space constraints or color figure charges

- Immediate publication on acceptance

- Inclusion in PubMed, CAS, Scopus and Google Scholar

- Research which is freely available for redistribution 